\title{
Regulatory Affairs
}

National Cancer Institute

\section{Source}

National Cancer Institute. Regulatory Affairs. NCI Thesaurus. Code C18995.

The business of a regulatory agency. 